\title{
A disturbance decoupling nonlinear control law for variable speed wind turbines
}

\author{
Thomsen, Sven Creutz; Poulsen, Niels Kjølstad
}

Published in:

IEEE Mediterranean Confrence on Control and Automation (MEDCON07)

Link to article, DOI:

10.1109/MED.2007.4433869

Publication date:

2007

Document Version

Publisher's PDF, also known as Version of record

Link back to DTU Orbit

Citation (APA):

Thomsen, S. C., \& Poulsen, N. K. (2007). A disturbance decoupling nonlinear control law for variable speed wind turbines. In IEEE Mediterranean Confrence on Control and Automation (MEDCONO7) IEEE.

https://doi.org/10.1109/MED.2007.4433869

\section{General rights}

Copyright and moral rights for the publications made accessible in the public portal are retained by the authors and/or other copyright owners and it is a condition of accessing publications that users recognise and abide by the legal requirements associated with these rights.

- Users may download and print one copy of any publication from the public portal for the purpose of private study or research.

- You may not further distribute the material or use it for any profit-making activity or commercial gain

- You may freely distribute the URL identifying the publication in the public portal 


\title{
A disturbance decoupling nonlinear control law for variable speed wind turbines
}

\author{
S.C. Thomsen*, N.K. Poulsen* \\ *Informatics and Mathematical Modelling, Build. 321, Technical University of Denmark, Kgs. Lyngby, Denmark
}

\begin{abstract}
This paper describes a nonlinear control law for controlling variable speed wind turbines using feedback linearization. The novel aspect of the control law is its ability to decouple the effect of wind fluctuations. Furthermore, the transformation to feedback linearizable coordinates is chosen intelligently so that the majority of the system structure is invariant under the transformation. Consequently the physical interpretation is preserved. The method assumes that the effective wind speed and acceleration are estimated from measurements on the wind turbine. The performance of the control is compared to that of a LQG controller using a specific wind turbine and wind model.
\end{abstract}

\section{INTRODUCTION}

As a result of increasing environmental concern, more and more electricity is being generated from renewable sources. In Denmark, wind turbines have experienced a broad popularity since the introduction in the early 1970s, now covering 20 percent of the Danish energy consumption. Ongoing research focuses on increasing the efficiency of the individual turbines and the parks of wind turbines as entities.

The basic operation of a wind turbine can be explained briefly: Wind turbines harness the power of the wind by using rotors, fitted with aerodynamic blades, to turn a drive shaft. The drive shaft rotates inside a generator which will then produce electricity. The type of wind turbine which is considered in this paper is horizontal axis pitch regulated wind turbines with variable speed asynchronous generators. 'Variable speed' simply means that it is possible to vary the relative speed of the generator, compared to the speed/frequency of the electrical grid. In effect, changing the relative speed changes the generator torque and thereby provides a useful control parameter.

Modern turbines rely on complex control systems to maximize efficiency and ensure safe operation. Control of wind turbines can be divided into three levels [1]. On the top level is the supervisory control, which monitors the turbine and wind resource in order to determine when to startup the turbine, shut down the turbine and shift between control strategies. On the middle level is the turbine controller which controls the blade pitch angle and generator torque. The turbine controller also controls the yaw of the nacelle, so that the nacelle points into the wind. However, this is a relatively slow motion compared to the dynamics of the turbine and hence is not of particular interest. The lowest level constitutes controllers for the power electronics, internal generator and pitch actuator.

In the present work the turbine controller has been considered. The primary objective of the turbine controller is to extract as much energy from the wind as possible.
However, practical limitations of the generator and the turbine mechanics prevent such operation. Consequently the objective is changed according to the wind speed as illustrated in Fig. 1. Region IV is considered in the present work. In this region, the power available in the wind exceeds the limit for which the generator and the turbine mechanics have been designed. Hence, to avoid failure, the rotational speed and output power should be kept constant at nominal values. A description of the other regions can be found in [2].

Much research has been focused at designing efficient turbine controllers. Classical methods (eg. PID control) have been widely applied in real life whereas advanced modern control has been applied to a lesser extend [3]. However, a huge body of literature concerning control of wind turbines with modern methods exist. Most prominent is the study on linear optimal and/or robust methods such as LQ, $\mathcal{H}_{2}$ and $\mathcal{H}_{\infty}$. Reference [4] gives a comparison between some of these advanced methods and classical control. Recently, nonlinear control of wind turbines has been the interest of several scientists. This ranges from well established methods such as sliding mode control and feedback linearization ([5], [6] and [7]) to methods such as neural networks and wavelets [8].

The main contribution of the present work is the derivation of a nonlinear control law which - ideally cancels the nonlinearities of the system and decouples the fluctuations in the wind speed from the system states. Feedback linearization is utilized and it is shown that the nonlinearities can be targeted intelligently ie. only the subspace in which the nonlinearities occur is compensated. The design is based on a medium complexity

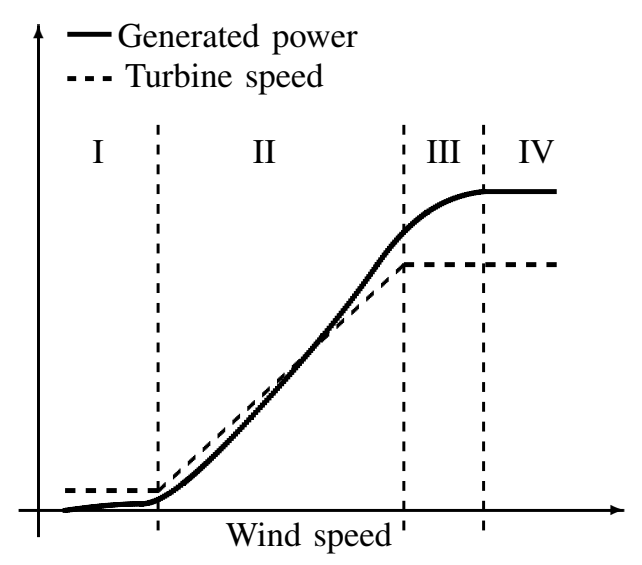

Fig. 1. Control regions for a turbine controller. The control law presented in this paper is designed for region IV. 


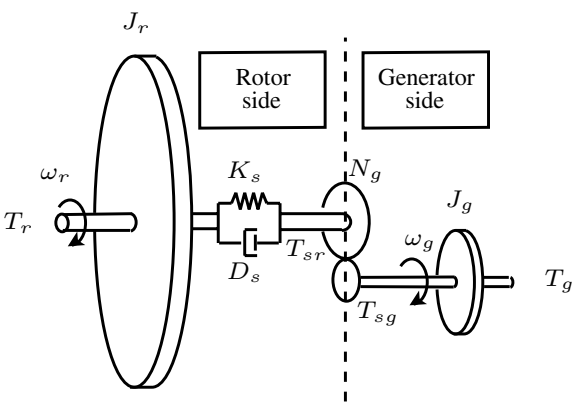

Fig. 3. Schematic of the wind turbine mechanics

turbine model, which includes dynamic characteristics commonly discarded when dealing with nonlinear wind turbine control.

\section{WIND TURBINE MODEL}

This section presents the dynamic model of a pitch controlled variable speed wind turbine. The wind turbine characteristics which are incorporated in the model are aerodynamics, turbine mechanics, generator dynamics and pitch actuator dynamics. Sub-models describing these characteristics will in the following be considered individually. The interconnections between these sub-models is illustrated in Fig. 2. Detailed descriptions of the subblocks, and the associated mathematical models, are given in the following sections.

\section{A. Mechanics}

Fig. 3 shows a schematic of the wind turbine mechanics. The turbine is split into two parts separated by the transmission: The rotor side and the generator side. The inertia on the rotor side $J_{r}$ and generator side $J_{g}$ are illustrated by the leftmost and the rightmost disk respectively. The shaft (drive train) connecting the rotor to the transmission is subject to immense torques that cause it to deflect. The shaft is appropriately modelled as a damped spring. This is illustrated by the damping $D_{s}$ and the spring constant $K_{s}$. The gear ratio $N_{g}$ is illustrated by the disks in the middle. On the left, the turbine is exited by the rotor torque $T_{r}$ and on the right the generator torque $T_{g}$. The torques $T_{s r}$ and $T_{s g}$ are the torques on each side of the transmission, which are related by the gear ratio:

$$
T_{s g}=\frac{T_{s r}}{N_{g}}
$$

The dynamics on the rotor side and generator side are described by (2) and (3).

$$
\begin{aligned}
& \dot{\omega}_{r} J_{r}=T_{r}-T_{s r} \\
& \dot{\omega}_{g} J_{g}=T_{s g}-T_{g}
\end{aligned}
$$

where $\omega_{r}$ and $\omega_{g}$ are the rotational speeds on the rotor side and generator side respectively. Introducing a variable $\delta$ [rad] describing the deflection of the drive shaft, leads to the following equation describing the twist of the flexible shaft:

$$
T_{s r}=D_{s} \dot{\delta}+K_{s} \delta
$$

where

$$
\dot{\delta}=\omega_{r}-\frac{\omega_{g}}{N_{g}}
$$

\section{B. Aerodynamics}

The aerodynamic blades on the rotor converts the kinetic energy of the wind into mechanical energy, effectively providing the torque $T_{r}$ on the rotor:

$$
T_{r}=\frac{P_{r}}{\omega_{r}}
$$

where the power $P_{r}$ is given by the following relation [9]:

$$
P_{r}=\frac{1}{2} \rho \pi R^{2} v^{3} c_{p}(\lambda, \theta)
$$

$\rho$ is the air density, $R$ the wing radius and $v$ the effective wind speed. $c_{p}$ is the power coefficient which is a function of the blade pitch angle $\theta$ and the tip speed ratio $\lambda$ defined below

$$
\lambda=\frac{v}{v_{t i p}}=\frac{v}{R \omega_{r}}
$$

The power coefficients $c_{p}$ for wind turbines are commonly attained utilizing blade element momentum (BEM) theory. A three dimensional plot of the power coefficient for a specific test turbine is shown in Fig. 4.

Fig. 5 shows iso-power curves given the power coefficient in Fig. 4. The curves are calculated for the rotational speed fixed at the nominal value $\omega_{r, n o m}=4.3 \mathrm{rad} / \mathrm{s}$. Given the nominal power $P_{r, \text { nom }}=225 \mathrm{~kW}$, Fig. 5 shows that it is nessesary to decrease the efficiency of the blades when the effective wind speed exceeds approx. $v=11 \mathrm{~m} / \mathrm{s}$ (ie. working in region IV). In the present

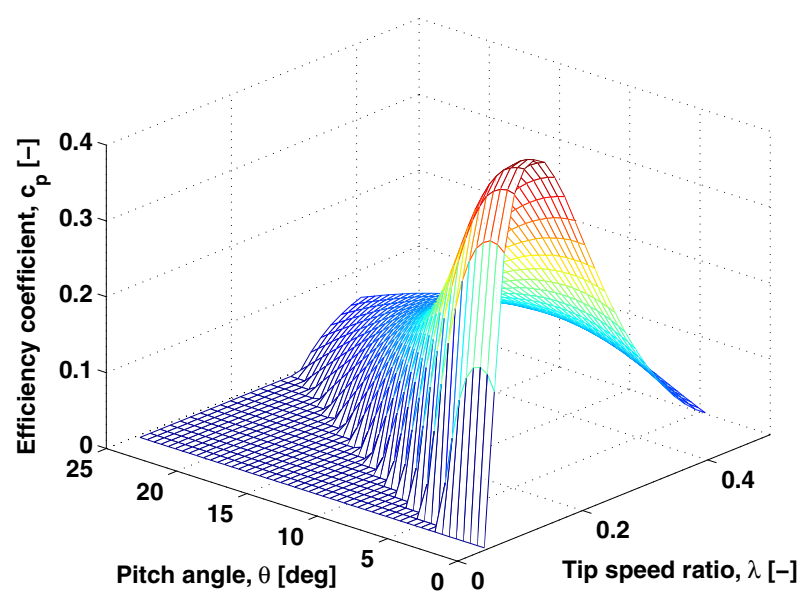

Fig. 4. The power coefficient $c_{p}$ for the blades on the test turbine. Negative values have been truncated. 
The state vector $x$ and input vector $u$ are defined as:

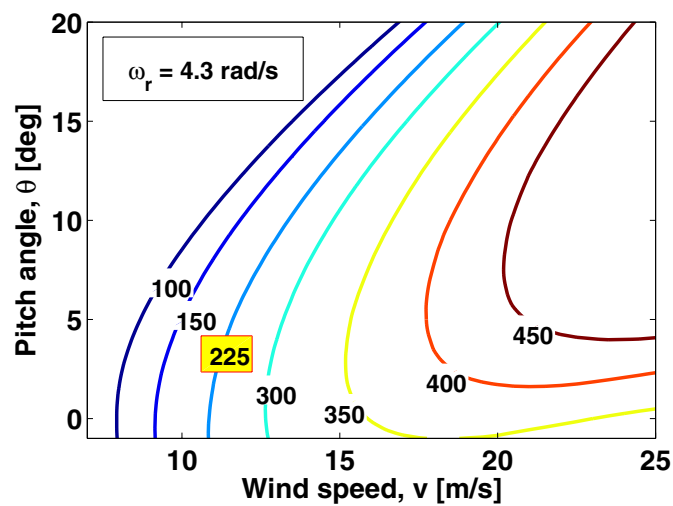

Fig. 5. Iso-power curves $(\mathrm{kW})$ based on the power coefficient for the test turbine. The curves are generated at the nominal rotational speed.

work this is accomplished by increasing the pitch. Note that decreasing the pitch will also decrease the energy capture (socalled stall regulation [3]).

\section{Generator dynamics}

The generator power is given by:

$$
P_{e}=T_{g} \omega_{g}
$$

As mentioned, the generator torque $T_{g}$ can be controlled, however, it cannot be changed instantaneously. The dynamic response of the generator has therefore been modelled by a first order linear model with time constant $\tau_{T}$ :

$$
\dot{T}_{g}=-\frac{1}{\tau_{T}} T_{g}+\frac{1}{\tau_{T}} T_{g, r}
$$

$T_{g, r}$ is the reference and one of the control inputs for the wind turbine.

\section{Pitch actuator}

The pitch of the blades is changed by a hydraulic/mechanical actuator. A simplified model of the dynamics is presented by the following first order linear model:

$$
\dot{\theta}=-\frac{1}{\tau_{\theta}} \theta+\frac{1}{\tau_{\theta}} \theta_{r}
$$

$\theta_{r}$ is the reference and the other control input for the wind turbine.

\section{E. Nonlinear state space description}

Combining all the previous equations results in the following nonlinear state space model affine in the control variable.

$$
\begin{aligned}
\dot{x}= & f(x, v)+G u \\
= & {\left[\begin{array}{c}
\frac{P_{r}\left(x_{1}, x_{4}, v\right)}{x_{1} J_{r}}-\frac{x_{1} D_{s}}{J_{r}}+\frac{x_{2} D_{s}}{J_{r} N_{g}}-\frac{x_{3} K_{s}}{J_{r}} \\
\frac{x_{1} D_{s}}{J_{g} N_{g}}-\frac{x_{2} D_{s}}{N_{g}^{2} J_{g}}+\frac{x_{3} K_{s}}{N_{g} J_{g}}-\frac{x_{5}}{J_{g}} \\
x_{1}-\frac{x_{2}}{N_{g}} \\
-\frac{1}{\tau_{\theta}} x_{4} \\
-\frac{1}{\tau_{T}} x_{5}
\end{array}\right] } \\
+ & {\left[\begin{array}{cc}
0 & 0 \\
0 & 0 \\
0 & 0 \\
\frac{1}{\tau_{\theta}} & 0 \\
0 & \frac{1}{\tau_{T}}
\end{array}\right] u }
\end{aligned}
$$

$$
\begin{aligned}
x & =\left[\begin{array}{lllll}
\omega_{r} & \omega_{g} & \delta & \theta & T_{g}
\end{array}\right]^{T} \\
u & =\left[\begin{array}{ll}
\theta_{r} & T_{g, r}
\end{array}\right]^{T}
\end{aligned}
$$

\section{WIND MODEL}

Knowing the effective wind speed $v$ and its time derivatives, makes it possible to compensate for wind speed fluctuations. However, the effective wind speed is an abstract quantity which describes the wind field experienced by the entire rotor disk. It is therefore not possible to obtain $v$ directly from measurements.

In this work we therefore assume that the effective wind speed is described by a suitable stochastic model and that the states of this model can be estimated from measurements on the wind turbine. In the derivation of the control law in section IV, only minor assumptions on this wind model will be made, however for the simulations in section $\mathrm{V}$ a specific wind model will be used. Both the general model and the simulation model is introduced in this section.

\section{A. General wind model}

The control law presented in this paper allows for a general class of wind models. Therefore, to proceed in a general fashion it is assumed that the wind is described by the following finite dimensional nonlinear state space model:

$$
\dot{w}=\alpha(w)+\beta(w) e
$$

where $e$ is a scalar stochastic process and the first element of $w$ is equal to the effective wind speed ie.

$$
w_{1}=v
$$

Additionally it is required that the relative degree between $w_{1}$ and $e$ is larger than one (ie. $e$ does not appear in $\dot{w}_{1}$ ). The reason for this requirement is justified in section IV. The wind turbine model (12) is trivially augmented with the wind model (16), the resulting system is written in condenced form below:

$$
\begin{aligned}
\dot{w} & =\alpha(w)+\beta(w) e \\
\dot{x} & =f\left(x, w_{1}\right)+G u
\end{aligned}
$$

\section{B. Simulation wind model}

The simulations in section $\mathrm{V}$ are based on a specific wind model which has been proposed in [2] and [10]. It is based on the observation that the effective wind speed can be described as a superposition of a slowly varying average wind speed $v_{m}$ superimposed by a rapidly varying turbulent wind speed $v_{t}$ ie. $v=v_{m}+v_{t}$. The mean wind speed is assumed measurable whereas the turbulent wind speed is described by a non-rational power spectrum which depends on $v_{m}$. For a fixed mean wind speed this spectrum can be reasonably approximated by a linear second order stochastic model driven by Gaussian distributed white noise [2]. Consequently, the effective 
wind speed can be described by the following state space model

$$
\left[\begin{array}{l}
\dot{w}_{1} \\
\dot{w}_{2}
\end{array}\right]=\left[\begin{array}{cc}
0 & 1 \\
-a_{1} & -a_{2}
\end{array}\right]\left[\begin{array}{c}
w_{1}-v_{m} \\
w_{2}
\end{array}\right]+\left[\begin{array}{l}
0 \\
k
\end{array}\right] e,
$$

where $\left[\begin{array}{ll}v & \dot{v}\end{array}\right]^{T}=\left[\begin{array}{ll}w_{1} & w_{2}\end{array}\right]^{T}$ and $e \in N\left(0,1^{2}\right)$. The parameters $a_{1}, a_{2}$ and $k$ are scheduled according to $v_{m}$.

\section{NONLINEAR CONTROL LAW}

This section will show that it is possible to compensate for the nonlinearities in the system and decouple the wind fluctuations. The tool with which the goal is accomplished is MIMO feedback linearization. Feedback linearization theory provides systematic methods with which nonlinear feedback compensators can be designed. This compensator is used in an inner loop which renders the system linear. Consequently, a linear controller can be designed for the compensated system. For a thorough review of the subject, readers are referred to [11] and [12]. Only input-state linearization will be considered in this paper.

\section{A. Review of input-state linearization}

This section provides the basics of input-state linearization. Consider a nonlinear system affine in the control variable

$$
\dot{x}=f(x)+G(x) u=f(x)+\sum_{i=1}^{m} g_{i}(x) u_{i},
$$

where $x \in \mathbb{R}^{n}, u \in \mathbb{R}^{m}$ and $f(x), g_{1}(x), \ldots, g_{m}(x)$ are smooth vector fields defined on an open subset of $\mathbb{R}^{n}$. Assume that a diffeomorphic transformation

$$
\left[\begin{array}{l}
\eta \\
\xi
\end{array}\right]=T(x), \quad \eta \in \mathbb{R}^{n-r}, \quad \xi \in \mathbb{R}^{r}
$$

exists, which brings the system to the following form:

$$
\begin{aligned}
\dot{\eta} & =\phi(\eta, \xi) \\
\dot{\xi} & =A \xi+B\left(f_{\xi}(x)+G_{\xi}(x) u\right)
\end{aligned}
$$

The input state relation between $u$ and $\xi$ is input-state linearizable, since the following nonlinear feedback

$$
u=G_{\xi}(x)^{-1}\left(-f_{\xi}(x)+\nu\right),
$$

renders the relation between $\nu$ and $\xi$ linear:

$$
\dot{\xi}=A \xi+B \nu
$$

The states $\eta$ are readily decoupled from the input-state description (26) and are therefore denoted internal dynamics. If $r=n$ there are no internal dynamics and the system is said to be full-state linearizable.

A diffeomorphism which brings the system to feedback linearizable coordinates can be obtained through the solution to a set of partial differential equations [11]. Intuitively, this corresponds to finding $m$ input-output relations with a total relative degree of $r=n$. This leads directly to a system description where the pair $(A, B)$ is a canonical form. This is not necessarily the most intelligent choice since any useful structural properties are not considered. Essentially it takes all system with the same dimensions to the same canonical form. As shown in the next section we avoid such a transformation by exploiting that the nonlinearities only enter in a small part of the system description.

\section{B. Input-state linearization applied to the wind turbine}

It is interesting to note that when a diffeomorphic transformation confines the disturbances of the system to the internal dynamics, the input-state description (26) is effectively decoupled from these disturbances. In application to the augmented wind turbine model (18)-(19) we therefore seek a diffeomorphism which confines the stochastic process $e$ to the internal dynamics such that the stochastic fluctuations can be decoupled from the feedback linearized system.

The nonlinearities of the turbine are confined to a subset of the system description. This structural property is easily exploited by choosing the following diffeomorphic transformation

$$
\left[\begin{array}{c}
w \\
T(w, x)
\end{array}\right]=\left[\begin{array}{c}
w \\
x_{1}-x_{1,0} \\
L_{f} x_{1} \\
x_{2}-x_{2,0} \\
x_{3}-x_{3,0} \\
x_{5}-x_{5,0}
\end{array}\right]=\left[\begin{array}{c}
w \\
\xi
\end{array}\right]
$$

which essentially only touches the input-output channel between $\theta$ and $\omega_{r} . x_{*, 0}$ denotes stationary values. The transformed system takes the following form:

$$
\begin{aligned}
\dot{w} & =\alpha(w)+\beta(w) e \\
\dot{\xi} & =A \xi+B\left(f_{\xi}(x, w)+G_{\xi}(x, w) u+\right)
\end{aligned}
$$

where

$$
f_{\xi}(x, w)=\left[\begin{array}{c}
\bar{L}_{\alpha, f}^{2} x_{1} \\
0
\end{array}\right] \quad G_{\xi}(x, w)=\left[\begin{array}{cc}
\bar{L}_{\beta, g_{1}} \bar{L}_{\alpha, f} x_{1} & 0 \\
0 & 1
\end{array}\right]
$$

By the operator $\bar{L}$ we associate the following simple extension of the usual Lie derivative:

$$
\bar{L}_{f, g} h(x, y) \equiv\left[\begin{array}{ll}
\frac{\partial h(x, y)}{\partial x} & \frac{\partial h(x, y)}{\partial y}
\end{array}\right]\left[\begin{array}{l}
f(x, y) \\
g(x, y)
\end{array}\right],
$$

As indicated in the transformed system description (28)(29), the stochastic process $e$ is restricted to the internal dynamics. This is a consequence of the requirement that the relative degree between $e$ and $w_{1}$ must be larger than 1.

By inspection it can be verified that $f_{\xi}$ and $G_{\xi}$ only depend on the wind model through the vector $w_{s}=$ $\left[w_{1} \frac{\partial w_{1}}{\partial w} \alpha(w)\right]^{T}=\left[\begin{array}{ll}v & \dot{v}\end{array}\right]^{T}$ ie.

$$
f_{\xi}(x, w) \equiv f_{\xi}\left(x, w_{s}\right) \quad G_{\xi}(x, w) \equiv f_{\xi}\left(x, w_{s}\right)
$$

Consequently, the effective wind speed and acceleration need to be known to compensate for the nonlinearities and the wind fluctuations. 
The compensated wind turbine system is given by:

$$
\begin{aligned}
\dot{w}= & \alpha(w)+\beta(w) e \\
\dot{\xi}= & A \xi+B \nu \\
= & {\left[\begin{array}{ccccc}
0 & 1 & 0 & 0 & 0 \\
0 & 0 & 0 & 0 & 0 \\
\frac{D_{s}}{J_{g} N_{g}} & 0 & -\frac{D_{s}}{J_{g} N_{g}^{2}} & \frac{K_{s}}{J_{g} N_{g}} & -\frac{1}{J_{g}} \\
1 & 0 & -\frac{1}{N_{g}} & 0 & 0 \\
0 & 0 & 0 & 0 & -\frac{1}{\tau_{T}}
\end{array}\right] \xi } \\
& +\left[\begin{array}{cc}
0 & 0 \\
1 & 0 \\
0 & 0 \\
0 & 0 \\
0 & \frac{1}{\tau_{T}}
\end{array}\right] \nu
\end{aligned}
$$

Hence, the variations in the wind speed have been completely decoupled from the closed loop wind turbine dynamics. Naturally, decoupling can only be attained perfectly given a perfect model and measurements. Although this requirement is not satisfied in real life, section $\mathrm{V}$ shows that good performance can still be attained.

\section{Linear controller for compensated system}

The infinite horizon linear quadratic (LQ) controller has been chosen as the underlying linear control scheme in the nonlinear controller setup. Due to the inherent disturbances caused by non-perfect knowledge of the system, it is essential that integral control is utilized to robustify the system and minimize bias. The final design will be denoted LQI (linear quadratic integral). Since we are interested in stabilizing $P_{e}=x_{2} x_{5}$ and $\omega_{g}=x_{2}$ the following first order approximation is introduced:

$$
\begin{aligned}
y & =C \xi \\
& =\left[\begin{array}{ccccc}
0 & 0 & T_{g, \text { nom }} & 0 & \omega_{g, n o m} \\
0 & 0 & 1 & 0 & 0
\end{array}\right] \bar{\xi}
\end{aligned}
$$

This allows for the following trivial augmentation of the system

$$
\left[\begin{array}{c}
\dot{\xi} \\
\dot{z}
\end{array}\right]=\left[\begin{array}{cc}
A & 0 \\
C & 0
\end{array}\right]\left[\begin{array}{c}
\xi \\
z
\end{array}\right]+\left[\begin{array}{c}
B \\
0
\end{array}\right] \nu
$$

the first order approximation is reasonable since the closed loop system is designed so that the $P_{e}$ and $\omega_{g}$ are close to their nominal values.

The LQI controller gain is obtained by minimizing cost (38) subject to (37).

$$
J=\int_{0}^{\infty}\left(\left[\begin{array}{l}
\xi \\
z
\end{array}\right]^{T} Q\left[\begin{array}{l}
\xi \\
z
\end{array}\right]+\nu^{T} R \nu\right) d t
$$

Figure 6 shows a block diagram of the total setup for the nonlinear controller.

\section{Simulations}

This section presents simulations which test the performance of the nonlinear control algorithm. The nonlinear controller is compared to a LQG controller (also with integral action). The cost function used in the LQG design is almost equal to the one used in the nonlinear controller. The only difference is that $\theta$ is weighted instead of $\dot{\omega}_{r}$

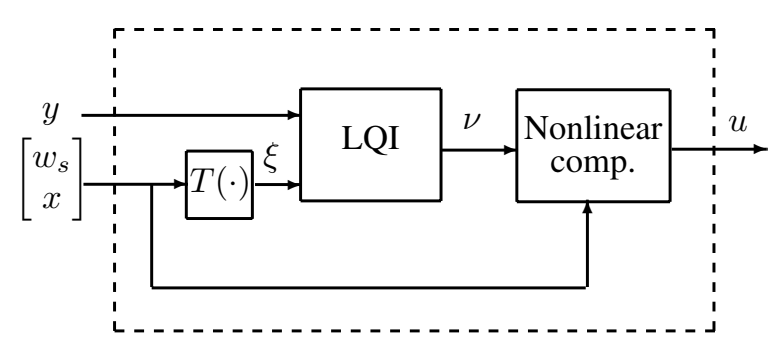

Fig. 6. Block diagram of the nonlinear controller

due to the nonlinear state transformation. The wind model (20) has been used to provide the wind input to the turbine model.

Given ideal conditions (perfect model and full state information), the nonlinear control law is superior, since it effectively decouples the wind fluctuations. Perturbations have therefore been introduced to test the robustness of the controller.

\section{A. Model perturbations}

It is assumed that the physical output $y_{1}=P_{e}$ and the turbine states $x$ are measured. The measurements are perturbed with noise to reflect non-ideal knowledge of the system:

$$
y_{m}=\left[\begin{array}{c}
y_{1} \\
x
\end{array}\right]+\zeta
$$

Where $y_{m}$ is the measurement vector and $\zeta$ is the measurement noise. The noise signals $\zeta_{1}, \zeta_{2}, \ldots, \zeta_{6}$ are modelled as uncorrelated Gaussian distributed white noise signals. The standard deviation is set to $1 \%$ of the stationary values. An extended Kalman filter has been designed to supply the estimates. The control setup is seen in figure 7. To reflect a non-perfect knowledge of the power coefficient the 'true' $c_{p}$ values are chosen to be $95 \%$ of the ones used in the controller.

\section{B. Results}

Figure 8 shows the wind sequence used in the simulations. Fig. 9 shows the response of $P_{e}, \omega_{g}$ and $\delta$ when controlled by the nonlinear controller and the LQG controller. Fig. 10 shows the corresponding control signals.

The physical output $P_{e}$ is compensated almost equally well by the two control strategies. However, the LQG controller does this at the cost of considerably larger control activity in the generator and larger stress on the turbine

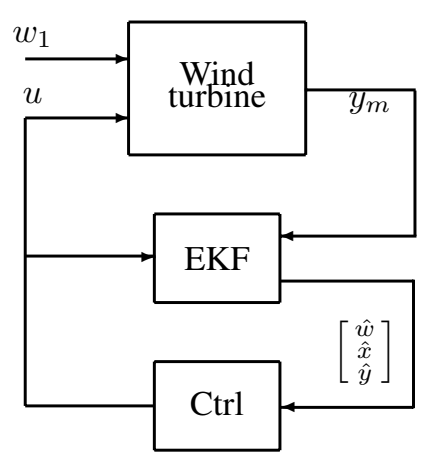

Fig. 7. Control setup with nonlinear controller and state estimator 


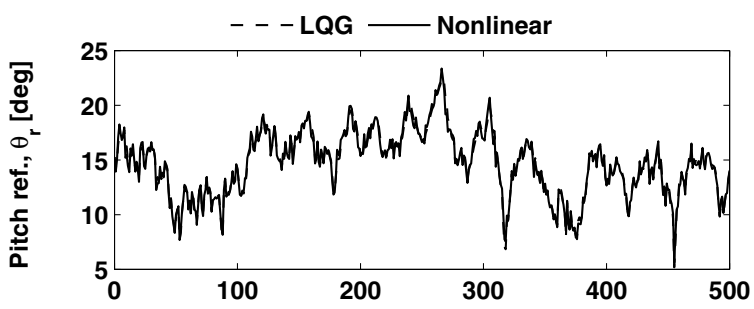

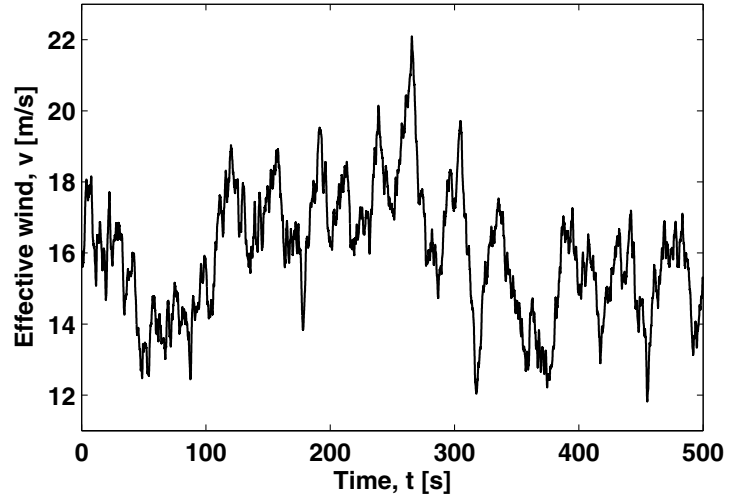

Fig. 8. Stochastic wind sequence used in simulations. The mean wind speed is $v_{m}=16 \mathrm{~m} / \mathrm{s}$.

mechanics $\left(\omega_{r}, \omega_{g}\right.$ and $\left.\delta\right)$. Hence, given the introduced perturbations, the nonlinear controller reduces the effect of the stochastic wind considerably better than the linear controller. As a final note it is worth mentioning that neither control strategy cause saturation of the actuators.

\section{CONCLUSION}

A nonlinear control law based on feedback linearization has been designed for variable speed wind turbines in the present work. The main advantage of the control law is its ability to decouple the fluctuations in the wind from the dynamics of the turbine. Furthermore, the nonlinear compensation does not take the system to the usual canonical form but conserves large parts of the
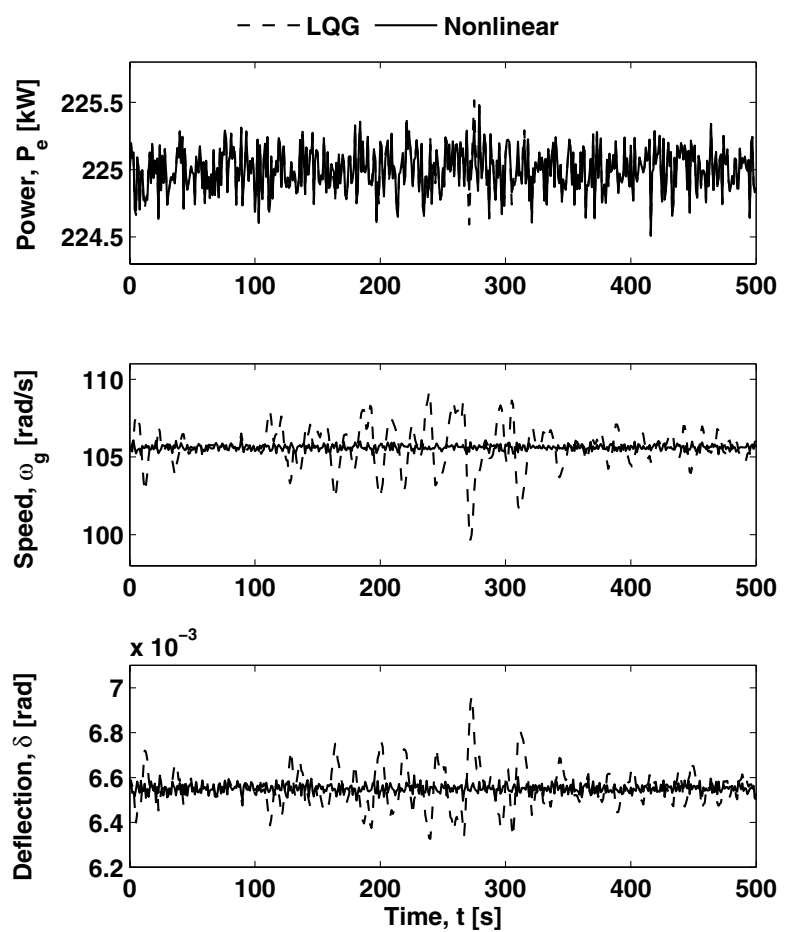

Fig. 9. Closed loop response associated with an LQG controller and the nonlinear controller

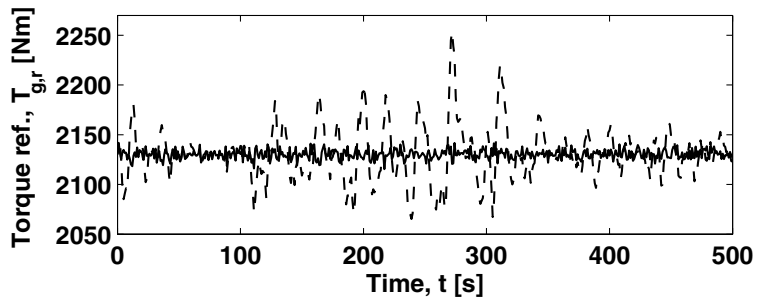

Fig. 10. Control signals from the LQG and nonlinear controller respectively.

original dynamics. The control law assumes knowledge of the wind, but is valid for a fairly general class of wind models. Simulations with system perturbations show that the control law attenuates the effect of wind fluctuations effectively although the decoupling properties are essentially compromised. Comparison with a LQG controller shows the advantage gained using nonlinear control as compared to linear control.

\section{REFERENCES}

[1] K. Johnson, L. Pao, M. Balas, and L. Fingersh, "Control of variable-speed wind turbines: standard and adaptive techniques for maximizing energy capture," Control Systems Magazine, IEEE, vol. 26, no. 3, pp. 70-81, 2006.

[2] M. Xin, "Adaptive extremum control and wind turbine control," Ph.D. dissertation, Informatics and Mathematical Modelling, Technical University of Denmark, 1997. [Online]. Available: http://www2.imm.dtu.dk/pubdb/p.php?2441

[3] T. Burton, D. Sharp, N. Jenkins, and E. Bossanyi, Wind Energy Handbook. Wiley, 2001.

[4] M. Grimble, "Horizontal axis wind turbine control: comparison of classical, lqg and h $\infty$ designs," Dynamics and Control, vol. 6, no. 2, pp. 143-161, 1996.

[5] B. Boukhezzar and H. Siguerdidjane, "Nonlinear control of variable speed wind turbines without wind speed measurement," $D e$ cision and Control, 2005 and 2005 European Control Conference. CDC-ECC '05. 44th IEEE Conference on, pp. 3456-3461, 2005.

[6] H. De Battista, R. Mantz, and C. Christiansen, "Dynamical sliding mode power control of wind driven induction generators," Energy Conversion, IEEE Transaction on, vol. 15, no. 4, pp. $451-457$, 2000.

[7] D. Leith and W. Leithead, "Implementation of wind turbine controllers," International Journal of Control, vol. 66, no. 3, pp. 349-380, 1997.

[8] M. Sedighizadeh, M. Kalantar, S. Esfandeh, and D. ArzaghiHarris, "Nonlinear model identification and control of wind turbine using wavenets," Control Applications, 2005. CCA 2005. Proceedings of 2005 IEEE Conference on, pp. 1057-1062, 2005.

[9] DNV/Risø, Guideline for design of wind turbines, 2nd ed DNV/Ris $\varnothing, 2002$.

[10] M. Xin, N. K. Poulsen, and H. Bindner, "Estimation of wind speed in connection to wind turbine," in Procededings for IASTED International Conference on Control, Cancun, Mexico, 1997, pp. $105-109$.

[11] A. Isidori, Nonlinear Control Systems, 3rd ed. Springer, 1995.

[12] J.-J. E. Slotine and W. Li, Applied Nonlinear Control. Prentice Hall, 1991. 